\title{
The Utilization of Hormonal Interaction during Stress to Get Better Performance
}

\section{Alaa A Elnour}

Department of Medical Lab Science, National Ribat University, Sudan

*Corresponding author: Elnour AA, Department of Medical Lab Science, National Ribat University, Sudan, Tel: 00966570727419; E-mail: alaa.a.elnour@outlook.com

Received date: March 23, 2018; Accepted date: April 24, 2018; Published date: April 27, 2018

Copyright: () 2018 Elnour AA. This is an open-access article distributed under the terms of the Creative Commons Attribution License, which permits unrestricted use, distribution, and reproduction in any medium, provided the original author and source are credited.

\begin{abstract}
Daily exposure to the stressor whether distress or eustress increase production of some hormones such as epinephrine, norepinephrine, cortisol, dopamine and serotonin and other. A group of them mainly function as excitatory and other function as inhibitory. However, the body reacts differently to distress and eustress while both secrete the same hormones. in case of flight to flight action that occur during distress, the interaction of these hormones enhances the body to be more focus on the surrounding environment, by stimulating senses memory, sight, hearing and other senses become sharper, however, long exposure to these hormones is dangerous on the heath. This body response should help to overcome adversity, whereas the body has different response it can be a positive or negative reaction, dealing with stress can be difficult, mindset and lifestyle have an enormous impact on stress which can help to adapt to stress reaction and utilization the hormonal interaction to get positive performance.
\end{abstract}

Keywords: Stress; Glucocorticoid; Catecholamines; Neurotransmitters; Performance

\section{Introduction}

Psychological impact has a huge effect on our physical state, whether if it happy or sad effect. Bad mental status is exhausting for both the body and mind; it could put our health at serious risk.

Sitting in traffic, late for an important meeting, watching the minutes tick away, or the positive event like receiving a promotion or raise at work, starting a new job, marriage, these things can cause an increase in heartbeats, breath quickens, and muscles tense up. All type of stress eustress or distress can lead to changes in the serum level of many hormones including glucocorticoids, catecholamines, growth hormone and prolactin.

the stress effect occurs in responding to adapt the individual to new circumstance, which is to increase mobilization of energy sources, It's a specific biochemical reaction that both humans and animals experience during intense stress or fear.

In response to a stressor, physiological changes are set into motion to help an individual cope with the stressor. However, chronic activation of these stress responses, which include the hypothalamicpituitary-adrenal axis and the sympathetic-adrenal-medullary axis, results in chronic production of glucocorticoid hormones and catecholamines [1].

The autonomic nervous system and hypothalamic-pituitary-adrenal (HPA) axis are two major systems that respond to stress; the HPA axis is a neuroendocrine system that mediates a stress response, is mediated by two different components: the sympathetic nervous system and the parasympathetic nervous system [2]. The classic fightor-flight reaction hormones are adrenaline, Cortisol, and Norepinephrine, also affects how organisms react to stress. There are other hormones induce during stress like the neurotransmitters dopamine and serotonin, as well as estrogen and testosterone [3].
The body goes through a number of changes: The fight or flight response to emergency or stress involves mydriasis, increased heart rate, and forcecontraction, vasoconstriction, bronchodilation, glycogenolysis, gluconeogenesis, lipolysis, sweating, decreased motility of the digestive system, secretion of the epinephrine and cortisol from the adrenal medulla, and relaxation of the bladder wall.

\section{The Function of Each Hormone}

Epinephrine, acetylcholine, and glutamate are mainly excitatory neurotransmitters while dopamine, norepinepinephrine, and GABA (gaba-amino-buteric-acid) are inhibitory [4].

\section{Catecholamines Biosynthesis}

Epinephrine and norepinephrine biosynthesis begin with sequential conversion of phenylalanine substrates, Tyrosine, DOPA, and to Dopamine. The dopamine convert into epinephrine and then the epinephrine convert into norepinephrine by cortisol-dependent enzyme. Any form of stress that increases the cortisol level stimulates epinephrine production [5].

Epinephrine, also known as adrenalin or adrenaline, which is increases the blood flow to muscles, the output of the heart, pupil dilation, and blood sugar [6].

Norepinephrine (NE), also called noradrenaline (NA) or noradrenalin, It's role is reorienting of attention and in cognitive flexibility. By enhances processing of sensory inputs, enhances attention, enhances formation and retrieval of both long term and working memory, and enhances the ability of the brain to respond to inputs by changing the activity pattern in the prefrontal cortex and other areas [7].

Cortisol the main glucocorticoid in humans, has been placed in this class because of its effects on the metabolism of the sugar glucose, where its primary function is to increase blood glucose levels by 
inducing production of additional glucose molecules to support the nutrient requirements of the CNS during stress [8]. ACTH is the principal regulator of cortisol production by the adrenal cortex.

Optimal levels of cortisol are needed not only to meet the body's physical needs but also for learning, memory, and cognitive performance [8].

Extensive evidence also indicates that epinephrine and glucocorticoids or stressful conditions that stimulate their release to enhance memory consolidation in human subjects when administered shortly before or after learning [9].

High stress levels during encoding and consolidation of emotional material involve increased amygdala and hippocampus activation [10].

Other hormones produced in the response to stress like dopamine and serotonin. They are major neuromodulators in the central nervous system.

The dopamine system is controlled by a set of mechanisms plays a central role in pleasurable reward behavior, inhibition of motor system, reward system, cognition, endocrine, nausea [11]. Evidence has contributed to the recognition of dopaminergic innervation as an important system for determining reactions to perturbations in environmental conditions [12].

Serotonin is an important neurotransmitter in the central nervous system where it modulates circuits involved in mood, cognition, movement, arousal, and autonomic function [13]. Serotonergic are involved in regulating mood and emotion, it also contributes to feelings of well-being and happiness [14,15].

This combination of hormones adrenaline, cortisol, norepinephrine and other like estrogen, testosterone, dopamine, and serotonin that produce during stress can help to perform better in situations where you are under pressure to do well. Extra oxygen is sent to the brain, increasing alertness. Enhance of memory, sight, hearing, and other senses become sharper. The interaction of these hormones improves body respond to difficult situations.

Moderate stress is healthy which can lead to cell growth in the brain's learning centers [16], but Long-term exposure to stress may lead to many deleterious consequences leading to various endocrine disorders. Also, stress leads to change in the clinical course or status of many endocrine conditions [17], and low levels of neurotransmitter may lead to conditions such as attention deficit hyperactivity disorder (ADHD), depression, and hypotension (very low blood pressure).

Stress effect is something that is giving you the strength and energy to overcome adversity, the body mobilizing its energy to get ready for challenge. But does it always give the ability to concentrate and react better (solve problems, plan ahead, understand others' actions, and control impulses)? How to control this power to get effective positive reaction? What the effect of long exposure to these hormones?

\section{How To Control the Stress Hormone And Utilize It For Better Performance For Daily Basis Activity?}

Stress is simply the body's response to changes that occur in the presence of the psychological reaction. Lifestyle and other environmental factors have a great impact on this reaction. Exercise training has potential health implications for dealing with chronic stress-related problems, chronic exposure to exercise training results in adaptations in the neuroendocrine system, such that there is a reduction in hormonal stress response to submaximal exercise and in many cases, reduced circulating basal stress hormone levels, but more research is necessary for this area [18]. Mindset is a significant factor in determining distress versus eustress. The positive mindset increases the chances of eustress and a positive response to stressors. Currently, the predominant mindset toward stress is that stress is debilitating. However, mindsets toward stress can be changed [19].

however long exposure to these hormones have a dangerous impact on the body considerable research has shown some hormones produced during psychological stress and can directly bind to cells to induce DNA, damage long-lasting consequences resulting in an increased potential for cellular transformation and/or tumor progression [20]. Avoid stress may be difficult so control and utilization of hormonal interaction of stress are important.

\section{Conclusion}

Positive emotional activities have been suggested as modifiers of neuroendocrine hormones involved in the classical stress response [21]. Studies have demonstrated that eustress and distress produce different responses in the neuroendocrine system, particularly dependent on the amount of personal control one feels over a stressor [22]. Findings of some study give partial support to the suggestion that personal control may attenuate sympathoadrenal activation and cardiovascular reactivity during stressful events [23]. Nerves are key to communicate within the body, that why the eustress considers positive and distress negatively, Lifestyle also contribute to stress hormones, regular exercise makes the neuroendocrine system adapt to stress response, which reduces the hormonal effect.

A lot of studies and research suggests methods to control and lower the stress hormones physiological methods, psychological or both. Nevertheless, almost nonexistent studies have shown how to utilize this power to get better performance.

\section{References}

1. Padgett DA, Glaser R (2003) How stress influences the immune response. Trends in Immunology 24: 444-8.

2. Jänig W (1989) Autonomic nervous system. In Human physiology. Springer, Berlin, Heidelberg; pp: 333-370.

3. Klein S (2013) Adrenaline, cortisol, norepinephrine: the three major stress hormones, explained. The Huffington Post.

4. https://brainfoodbrainfood.com/category/neurotransmitter-pathwaysserotonin-dopamine-acetylcholine/

5. Bishop ML, Fody EP, Schoeff LE (2013) Clinical chemistry: principles, techniques, and correlations. Lippincott Williams \& Wilkins.

6. Rhoades RA, Bell DR (2012) Medical phisiology: Principles for clinical medicine. Lippincott Williams \& Wilkins.

7. Sara SJ (2015) Locus coeruleus in time with the making of memories. Current Opinion in Neurobiology 35: 87-94.

8. Stephens MA, Wand G (2012) Stress and the HPA axi: Role of glucocorticoids in alcohol dependence. Alcohol research: current reviews.

9. Bermúdez-Rattoni F (2007) Neural plasticity and memory: from genes to brain imaging. CRC Press.

10. Van Stegeren AH (2009) Imaging stress effects on memory: a review of neuroimaging studies. The Canadian J Psy 54: 16-27.

11. Ayano G (2016) Dopamine: Receptors, Functions, Synthesis, Pathways, Locations and Mental Disorders: Review of Literatures. J Ment Disord Treat 2: 2-4.

12. Pani L, Porcella A, Gessa GL (2000) The role of stress in the pathophysiology of the dopaminergic system. Molecular Psychiatry 5: 14. 
Citation: Elnour AA (2018) The Utilization of Hormonal Interaction during Stress to Get Better Performance. J Pharmacogenomics Pharmacoproteomics 9: 177. doi:10.4172/2153-0645.1000177

Page 3 of 3

13. Brindley RL, Bauer MB, Blakely RD, Currie KP (2017) Serotonin and serotonin transporters in the adrenal medulla: A potential hub for modulation of the sympathetic stress response. ACS Chemical Neuroscience 8: 943-954.

14. Hensler JG (2010) Serotonin in mood and emotion. InHandbook of Behavioral Neuroscience. Elsevier pp: 367-378.

15. Young SN (2007) How to increase serotonin in the human brain without drugs. J Psy \& Neuro 32: 394.

16. Kirby ED, Muroy SE, Sun WG, Covarrubias D, Leong MJ, et al. (2013) Acute stress enhances adult rat hippocampal neurogenesis and activation of newborn neurons via secreted astrocytic FGF2.

17. Ranabir S, Reetu K (2011) Stress and hormones. Indian J Endocrinology \& Metabolism 15: 18

18. Hackney AC (2006) Stress and the neuroendocrine system: the role of exercise as a stressor and modifier of stress. Expert Review of Endocrinology \& Metabolism 1: 783-792.
19. Ambriz MG, Izal M, Montorio I (2012) Psychological and social factors that promote positive adaptation to stress and adversity in the adult life cycle. J Happiness Studies 13: 833-848.

20. Flint MS, Baum A, Episcopo B, Knickelbein KZ, Liegey Dougall AJ, et al. (2013) Chronic exposure to stress hormones promotes transformation and tumorigenicity of 3T3 mouse fibroblasts. Stress 16: 114-121.

21. Berk LS, Tan SA, Fry WF, Napier BJ, Lee JW, et al. (1989) Neuroendocrine and stress hormone changes during mirthful laughter. The American J Med Sci 298: 390-396.

22. Dembroski TM (1983) Biobehavioral bases of coronary heart disease. Karger biobehavioral Medicine Ser.

23. Bohlin G, Eliasson K, Hjemdahl P, Klein K, Frankenhaeuser M (1986) Pace variation and control of work pace as related to cardiovascular, neuroendocrine, and subjective responses. Biological Psychology 23: 247-263. 